\title{
Longstanding Effect and Outcome Differences of Palatal Plate and Oral Screen Training on Stroke-Related Dysphagia
}

\author{
Mary Hägg ${ }^{*}, 1,2$ and Lita Tibbling ${ }^{3}$
}

\author{
${ }^{1}$ Speech \& Swallowing Centre, Department of Otorhinolaryngology, Hudiksvall Hospital, ${ }^{2}$ Research \& Development \\ Centre, Uppsala University/Gävleborg, ${ }^{3}$ Department of Otorhinolaryngology, Linköping University, Sweden
}

\begin{abstract}
Aim: This study aimed at evaluating (1) if the oral training effect on stroke related dysphagia differs between two different oral appliances, a palatal plate (PP) and an oral screen (OS), and (2) if the training effect remains at a late follow-up.

Methods: We included patients with stroke-related dysphagia at two different time periods: the first group of 12 patients studied in 1997- 2002 had to train with a PP, the other one of 14 patients studied in 2003-2008 had to train with an OS. All patients were evaluated by a swallowing capacity test (SCT), and by a self-assessed visual analogue scale (VAS) of water swallowing capacity at entry of the study, after 13 weeks of training, and at a late follow-up.

Results: At end of treatment the SCT had normalized in $33 \%$ of PP patients and in $71 \%$ of OS patients. There was a significant SCT improvement difference between the PP and OS groups in the period from baseline to late follow-up (p < 0.002) in favor of the OS group. VAS as tested at baseline and at end of treatment did not differ significantly between the two groups. Training with PP and with OS produced remaining improvement of SCT and of VAS as assessed at a late follow-up.

Conclusion: The outcome of OS training on SCT in patients with stroke-related dysphagia seems to be superior to PP training. The improvement as assessed with VAS did not differ between the two groups. Training with PP or OS gives a longstanding improvement of SCT and VAS.
\end{abstract}

Keywords: Dysphagia, oral screen, palatal plate, sensorimotor stimulation, stroke, swallowing capacity, VAS.

\section{BACKGROUND}

Swallowing problems following acute stroke are claimed to be present in about half of patients on admission [1-3].

Most stroke-related dysphagia problems during meals, e.g. aspiration, cough, drooling, food leakage, leakage through the nose, long meal times, and retention of food in the mouth, resolve over the first 7 days in one third of patients [4-6]. After one month, remaining dysphagia of around $10 \%$ is regarded to be more or less of chronic character $[7,8]$. In addition to so called compensatory techniques [9-13], dysphagia has been treated by oropharyngeal sensorimotor stimulation using oral appliances, such as a palatal plate (PP) [14] with improvement of dysphagia in 59\% [15], or as an oral screen (OS) [16] with improvement in $97 \%$ and with normalization in $63 \%$ [17]. Swallowing depends on a complicated interplay of sensory and motor functions involving a large number of muscle groups that has to achieve a proper balance. The PP and OS training appliances are aimed at triggering the four different sensory cranial nerves (V, VII, IX and X) of oropharynx involved in deglutition. Through the afferent paths the signals will be sent to the swallowing

*Address correspondence to this author at the Speech \& Swallowing Centre, Otorhinolaryngology Department, Hudiksvall Hospital, SE 82481 Hudiksvall, Sweden; Tel: +46 (0)650 92754; Fax: +46 (0)650 92412;

E-mail: mary.hagg@lg.se center in the brainstem and further on to the efferent motor paths of the five motor cranial nerves (V, VII, IX, X and XII) in order to rehabilitate patients afflicted with dysphagia after stroke [14-20].

The PP is designed to passive stimulate oropharyngeal tactile receptors and to actively and passively stimulate the buccinator mechanism, thus creating a negative intraoral pressure and initiating a swallowing reflex. The PP also elicits a constant search for unfamiliar objects by the tongue. It improves the contact between tongue and palate, raises the tip of the tongue, helps the tongue to contract upward and backward, activates the levator anguli oris muscles (VII), the zygomaticus minor and major muscles, and the buccinator muscles, thus indirectly improving oropharyngeal swallowing $[10,14,25]$. PP was the first to be applied to sensorimotor training in dysphagia studies $[14,15]$; the OS device has more recently been introduced $[16,17,21]$. The OS training (Fig. 1) stimulates oropharyngeal tactile receptors and motor function in order to create lip closure and to raise activity of the buccinator mechanism [14, 15, 21-24] supplied by facial nerves (VII), pharyngeal plexus including glossopharyngeal nerves (IX) and vagal nerves (X). When pulling the OS (Fig. 1), the intraoral mucous membranes supplied by trigeminal nerves (V) will be stimulated, and will trigger the entire natural activity chain from the lips to the pharynx. Tonus of the oral floor muscles $(\mathrm{V}, \mathrm{XII})$ increases, in turn causing tongue retraction (XII), in turn stimulating sensory parts of the anterior faucial arcs 
(IX), and soft palate (V, VII and X) [26]. The stylohyoid muscles and the posterior part of the digastric muscles (VII) are also activated. All these activities are prerequisites of attaining a negative intraoral pressure and of initiating a swallowing reflex $[14,17,20]$.

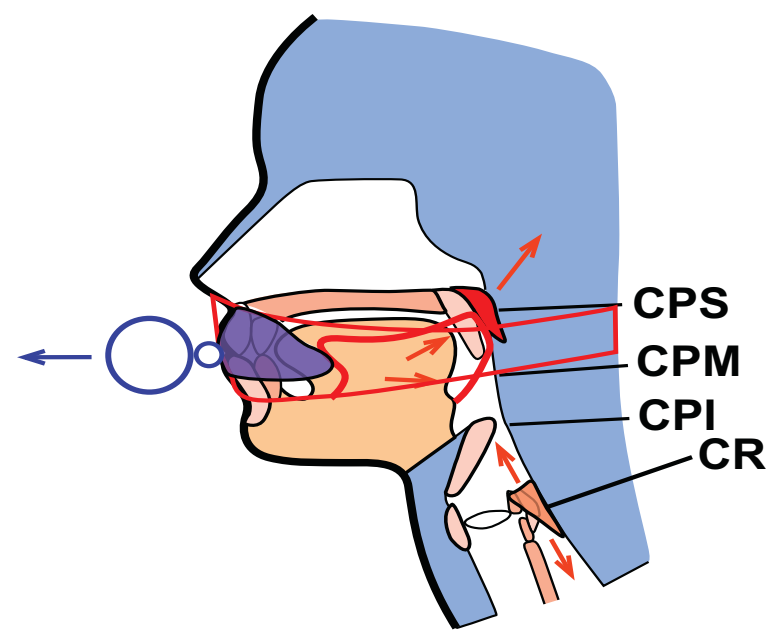

Fig. (1). OS training. The buccinator mechanism (marked with a line) involves $\mathrm{m}$. orbicularis oris, $\mathrm{m}$. buccinator, $\mathrm{m}$. constrictor pharyngeus superior (CPS), m. constrictor pharyngeus middle $(\mathrm{CPM}) ; \mathrm{m}$. constrictor pharyngeus inferior (CPI); $\mathrm{m}$. cricopharyngeus $(\mathrm{CR})=$ upper esophageal sphincter. The OS is placed predentally and stimulates the sensory input by touching the intra-oral membranes (V). When pulling the oral screen and pressing the lips (VII) the entire buccinator mechanism will be activated (VII, IX, X), tonus and contraction of the bottom of the mouth increase (V, XII) and act as a valve mechanism, thereby enhancing the intra-oral negative pressure. That in turn activates tongue retraction (XII), which in turn stimulates the sensory part of the anterior faucial arcs (IX), the soft palate (V, VII, X), and the intra-oral mucous membranes (V). Finally the stylohyoid and the digastric posterior muscles (VII) are activated.

Any comparison between the PP and OS modalities has not been performed before. Furthermore, it is unknown whether the different training modalities have remaining therapeutic effect. The aim of the current study was therefore to evaluate (1) if improvement of objective and subjective swallowing capacity differs between the PP and OS training methods in patients with stroke-related dysphagia, and (2) if any improving effect on objective and subjective swallowing capacity remains at a late follow-up. Fig. (1) designed by the authors $\mathrm{MH}$.

\section{METHODS}

\section{Study Design}

This study on two groups of patients with stroke-related dysphagia comprises evaluation of a 13-week training effect of PP in the period 1997-2002, and of OS in the period 20032008. The training effect was assessed with the water swallowing capacity test (SCT) and with a self-assessed scoring test (VAS) of water swallowing capacity before and after 13 weeks of training at home, and at a late follow-up. In addition, the study included a comparison of training effect between OS patients treated early and late after stroke occurrence. The tests were evaluated by one of the authors $(\mathrm{MH})$ at entry of the study and at late follow-up, and by a trained assistant after end of treatment. All data were blinded and sent to a statistical research centre.

\section{Study Population}

All 172 patients with a history of stroke-related dysphagia, as assessed by physicians in a stroke ward or by general practitioners, were referred to a swallowing treatment centre from 1997 to 2008. Inclusion criteria for this study were patients suffering from their first-ever stroke and a diagnosis of dysphagia according to referring physicians. Exclusion criteria were neurological diseases other than stroke, and inability to cooperate due to dementia. Of all patients referred to the swallowing treatment centre, two patients had other neurological diseases (amyotrophic lateral sclerosis, Huntington disease). Forty-one did not agree to study participation, and 50 patients died before attending the clinic. All remaining 79 patients agreed to participate and were initially enrolled. The following patients could not complete the study: 28 suffered a new stroke, 12 became unable to cooperate, and 13 died (4 due to another stroke, 9 due to cancer). Of the remaining 26 patients who signed a consent participation form, 12 patients (median age 69 years, range 46-82, 2 women and 10 men) were enrolled during the first time period and had to train with a PP, and 14 patients (median age 69 years, range 55-81, 7 women and 7 men) were enrolled during the second time period and had to train with an OS.

Oral sensorimotor training lasted for 13 weeks. Patients in the PP group started training at a median of 20 months (range 1-69) after stroke. In the OS group, 7 patients started training within 3 weeks after stroke and 7 patients began training on average 12 months (range 1-108) after stroke. During the pre-trial period, all patients had been given advice about optimal positioning during meals, adapted diet, and strict oral hygiene.

Cortical lesions were found on the left side in 14 patients (9/12 in the PP group), on the right side in 7 patients $(1 / 12$ in the PP group), and in the cerebellum in one patient (1/14 in the OS group). Bleeding was found in two patients from each group (one on each side in the PP group; one on the left cortical side and one in the brainstem in the OS group). Central facial paresis was found in 13 patients $(5 / 12$ in the PP group). Five patients had a history of aspiration pneumonia (4/12 in the PP group).

The long-term follow-up was performed on average 22 months (range 4-62) after completion of training in the PP group, and on average 13 months (range 5-21) after completion of training in the OS group.

\section{Training Design}

Training included a 13-week period at home in both groups. The PP group had an individual adjusted PP inserted in the upper jaw 3 times per day for 10-30 minutes before eating [15]. In the OS group, a standardized OS was inserted behind closed lips [17] for 5-10 s, 3 times per session before eating, 3 times daily. Verbal, practical, and written instructions about the training were given to the patients, relatives, or care assistants. The patient, family member, or assistant registered each training session at home in a training diary. Training compliance was supervised and the diary was checked up on at a speech and swallowing centre 
every three weeks during the entire training period. At these four check-up events, SCT and VAS were performed. All patients in the PP and OS groups attended all four check-up events during the training period.

\section{Palatal Plate (PP) Training}

The PP was composed of thin acrylic material with a metallic arrow running around the vestibular upper margins on which four vestibular small acrylic plates ("bumpers") were attached [14, 15]. These bumpers were supplied with stainless steel knobs aimed at passive stimulation of the upper lip and the buccinator mechanism [14, 15, 22-24]. For stimulation of the tip of the tongue, a mobile cube of stainless steel was attached to a dento-alveolar arch placed across the plate behind the incisors, in line with the canine teeth. For tongue base stimulation, a velar arch provided with three small pointed convexities in the midline and laterally, was placed on the plate close to the A-line (the border between the soft and hard palates). Six of the stroke patients had a maxillary denture of which a duplicate was fabricated. On this duplicate, stimulators were attached in order to function identical to an ordinary PP. Training with the PP plate inserted in the upper jaw during at least 10-30 minutes required the following exercises repeated three times, and three times a day before eating:

1. Stretching the upper lip caudally and holding it for one second.

2. Stretching the lips as far as possible in the 'ee' position and alternatively rounding the lips as tightly as possible.

3. Pulling the lips as far as possible alternately to the left and right side and holding each position for one second.

4. Elevating the tongue with an open mouth to reach the mobile cube, and moving it laterally, forward, and backward, as fast as possible.

5. Stretching the tongue laterally and upward against the vestibular stimulators, and licking the metallic knobs toward the middle line, thereafter repeating the same movements on the other side.

6. Opening the mouth and pushing the tongue against the front steel wire as hard as possible and holding this position for one second.

7. Pulling the tongue base backward against the back wire and holding this position for one second.

8. Trying to gargle as hard as possible.

9. Opening the mouth and pronouncing ' $\mathrm{k}$ ' as hard as possible with short pauses in between.

\section{Oral Screen (OS) Training}

The standardized OS is made of acrylic and supplied with a pulling loop. During training, the screen was inserted predentally behind closed lips and the patient had to sit in a chair or in the bed with the body in a strictly upright position, with support for the head. The patient had to hold the screen against the horizontal gradually increasing pulling pressure for 5-10 $\mathrm{s}$ while trying to resist the force by tightening the lips and pressing the head backward against a head rest $[16,17,21]$. The exercise was performed 3 times per session before eating, 3 times daily.

If the patient was unable to hold the oral screen, relatives or care assistants were instructed to assist with the traction at right angles to the patient's mouth.

\section{SCT}

The patient was instructed to sit upright and to swallow a glass of $150 \mathrm{ml}$ water as quickly as possible without pausing $[27,28]$. The time was recorded from onset of drinking until the last swallowing was completed. Remaining water in the glass was measured. When the patients could not swallow anything at all, or got an initial wrong-way swallowing, the SCT was graded as zero. The swallowing capacity was calculated as the amount of swallowed water $(\mathrm{ml})$ divided by time (s). A swallowing capacity index of $10 \mathrm{ml} / \mathrm{sec}$ is regarded as the lower limit of normal [27, 28].

\section{VAS}

The patient's self-assessed ability to swallow water at the SCT-test was scored from $0-100 \mathrm{~mm}$ on a VAS, where 0 is normal and 100 is total inability.

\section{Statistics}

Professional statisticians and a data manager from a clinical research center were involved from the outset in planning the PP study design, and later on the OS study design, according to good clinical practice (GCP), by use of a database to consolidate and analyze all collected data according to an initial protocol. Wilcoxon's signed rank test was used for comparison of data within groups and the Mann Whitney U-test was used for comparison of data between groups. A p-value $<0.05$ was considered significant.

\section{Ethical Consideration}

The local Ethics Committee for Human Research approved the study (Dnr 2004: M-435).

\section{RESULTS}

All patients had either a pathological SCT or a high VAS score at baseline: pathological SCT was found in $92 \%$ of PP patients and $93 \%$ of OS patients (Table 1, Fig. 2); pathological VAS was $100 \%$ in the both groups (Table 2, Fig. 3). After end of treatment, 33\% of PP patients and $71 \%$ of OS patients got a normalized SCT (Table 1, Fig. 2). There was a significant SCT improvement difference between the PP and OS groups in the period from baseline to late followup in favor of the OS group (Table 1). Median SCT did not change significantly from the end of training to late followup in the PP group (5.6 to $7.3 \mathrm{ml} / \mathrm{s}$; Table 1) nor in the OS group (12.6 to $11.8 \mathrm{ml} / \mathrm{s}$; Table $\mathbf{1})$.

Patients' self-assessed ability to swallow water according to VAS was significantly improved after training in the PP group and in the OS group; Table 2, Fig. 3). The change of improvement according to VAS between baseline and end of training did not differ significantly between the OS and PP groups (Table 2). VAS improvement between end of training and late follow-up was significantly pronounced in the OS group but not in the PP group (Table 2). However, any significant difference of VAS between the two groups was not found (Table 2). 
Table 1. Swallowing Capacity Test (SCT)

\begin{tabular}{|c|c|c|c|c|c|}
\hline Treatment Group & $\begin{array}{c}\text { PP }(n=12) \\
\text { Median SCT (Range) }\end{array}$ & p-Value & $\begin{array}{c}\text { OS ( }(n=14) \\
\text { Median SCT (Range) }\end{array}$ & p-Value & $\begin{array}{l}\text { Difference Between } \\
\text { PP and OS p-Value }\end{array}$ \\
\hline Baseline & $1.9(0.0-14.4)$ & & $0.9(0.0-10.0)$ & & $<0.784$ \\
\hline End of treatment & $5.6(0.0-18.8)$ & & $12.6(4.0-18.8)$ & & $<0.106$ \\
\hline Follow-up & $7.3(1.9-18.3)$ & & $11.8(5.5-27.7)$ & & $<0.02$ \\
\hline Change in SCT from baseline to end of treatment & $3.4(0.0-15.4)$ & $<0.001$ & $8.0(3.1-15.4)$ & $<0.001$ & $<0.02$ \\
\hline Change in SCT from baseline to follow-up & $5.0(1.3-14.7)$ & $<0.001$ & $11.6(4.1-19.9)$ & $<0.001$ & $<0.002$ \\
\hline Change in SCT from end of treatment to follow-up & $1.1(-14.1-7.0)$ & $<0.276$ & $2.4(-6.5-15.3)$ & $<0.153$ & $<0.434$ \\
\hline
\end{tabular}

Median SCT (ml/s) and ranges in the palatal plate (PP) and oral screen (OS) groups before treatment (baseline), at end of treatment, and at follow-up, changes in SCT between the three occasions, and improvement difference between the PP and OS groups. Statistical differences (p-value) within (Wilcoxon's signed rank test) and between (Mann Whitney Utest) the PP and OS groups, Diff $=$ difference, $-=$ decrease.

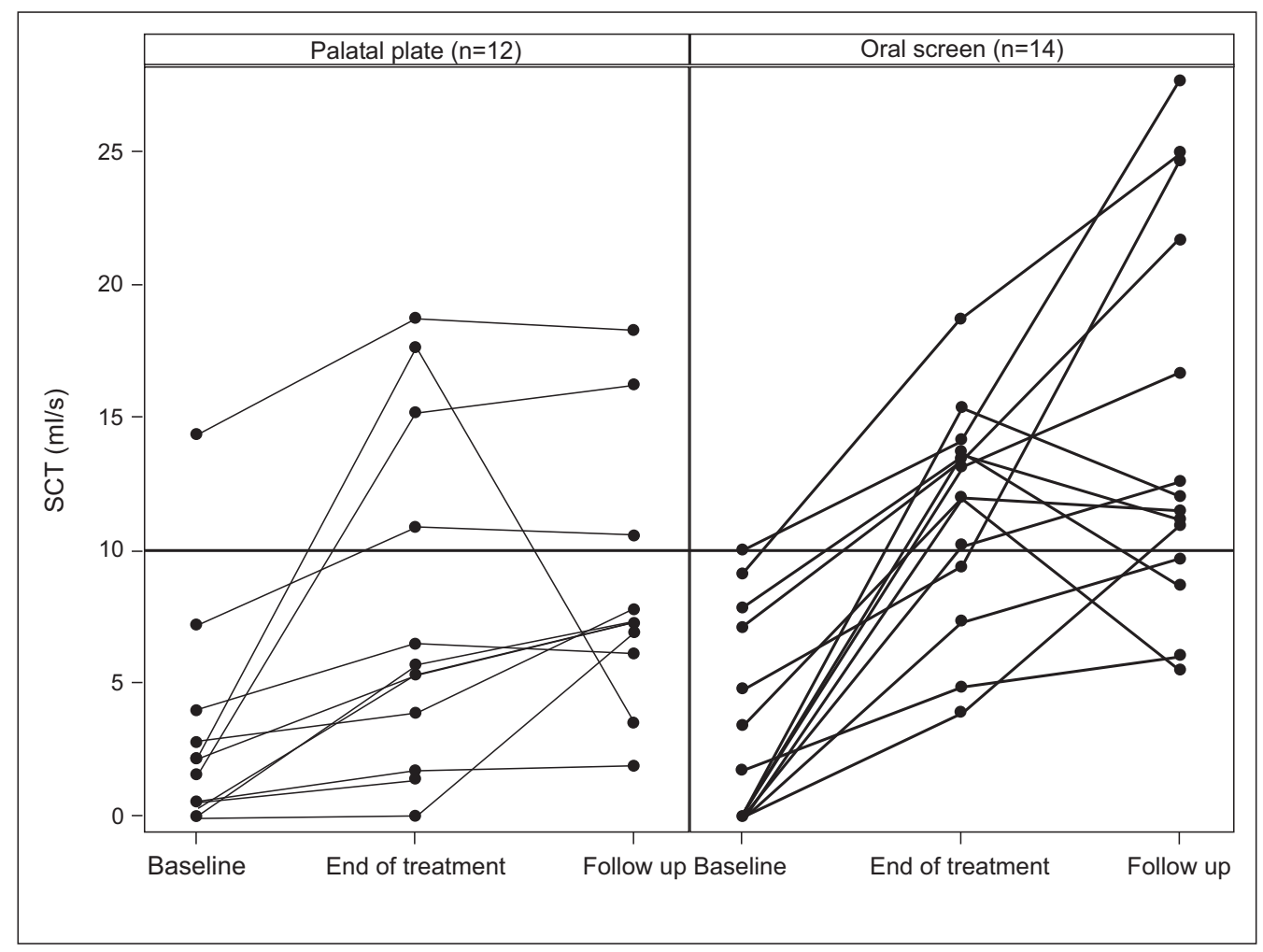

Fig. (2). Individual values on the swallowing capacity test (SCT). Individual values on the SCT (ml/sec) at baseline, end of treatment, and follow-up in stroke patients treated with a palatal plate (left) or with an oral screen (right).

When the OS group was separated according to duration of dysphagia from stroke attack to start of training, there was no difference in training results of SCT or VAS between the "Early" versus "Late" groups (Tables 3 and 4, Figs. 4, 5). Both OS groups showed a significant improvement of SCT and of VAS after end of treatment (Tables 3 and 4). No further improvement of SCT and of VAS was seen in the period from end of treatment to follow-up in the "Early" group, but on the other hand this was found with SCT in the "Late" group (Tables 3 and 4, Figs. 4, 5). At late follow-up SCT was normalized in all patients in the "Late" group, but only in $43 \%$ in the "Early" group (Table 3 and Fig. 4). This is also apparent in the individual values of SCT and VAS in the "Early" and "Late" OS groups (Figs. 4, 5).

\section{DISCUSSION}

Oral sensorimotor training with an OS was found to be significantly more successful than with a PP in improving swallowing capacity in patients with stroke-related dysphagia. The objective difference in training efficacy obtained between PP and OS may well depend on difference in triggering strength of the oropharyngeal sensorimotor system. The PP will improve function and strength in 58 muscles supplied by five different double-sided cranial nerves, and so will the OS as well. The difference between the two modalities is that the PP triggers muscles one by one $[14,15]$ whereas the OS triggers the entire natural chain of neuromuscular activity, that is the same as that initiating a swallow [16, 17] (Fig. 1). In contrast to an OS, a PP 
Table 2. Visual Analogue Scale (VAS)

\begin{tabular}{|c|c|c|c|c|c|}
\hline Treatment Group & $\begin{array}{c}\text { PP }(n=12) \\
\text { Median VAS (Range) }\end{array}$ & p-Value & $\begin{array}{c}\text { OS }(n=14) \\
\text { Median VAS (Range) }\end{array}$ & p-Value & $\begin{array}{l}\text { Difference Between } \\
\text { PP and OS p-Value }\end{array}$ \\
\hline Baseline & $83(50-100)$ & & $90(15-100)$ & & $<0.987$ \\
\hline End of treatment & $33(0-80)$ & & $15(0-80)$ & & $<0.146$ \\
\hline Follow-up & $15(0-80)$ & & $2(0-30)$ & & $<0.063$ \\
\hline Change in VAS from baseline to end of treatment & $-31(-90-0)$ & $<0.001$ & $-65(-100--5)$ & $<0.001$ & $<0.428$ \\
\hline Change in VAS from baseline to follow-up & $-40(-100-7)$ & $<0.002$ & $-80(-100--12)$ & $<0.001$ & $<0.383$ \\
\hline Change in VAS from end of treatment to follow-up & $-10(-40-80)$ & $<0.152$ & $-6(-74-3)$ & $<0.01$ & $<0.875$ \\
\hline
\end{tabular}

VAS (mm) in the palatal plate (PP) and oral screen (OS) groups before treatment (baseline), at end of treatment, and at follow-up, changes in median VAS between the different tes occasions, and improvement differences between the PP and OS groups. Statistical differences (p-value) within (Wilcoxon's signed rank test) and between (Mann Whitney U-test) the PP and OS groups, Diff $=$ difference, $-=$ decrease

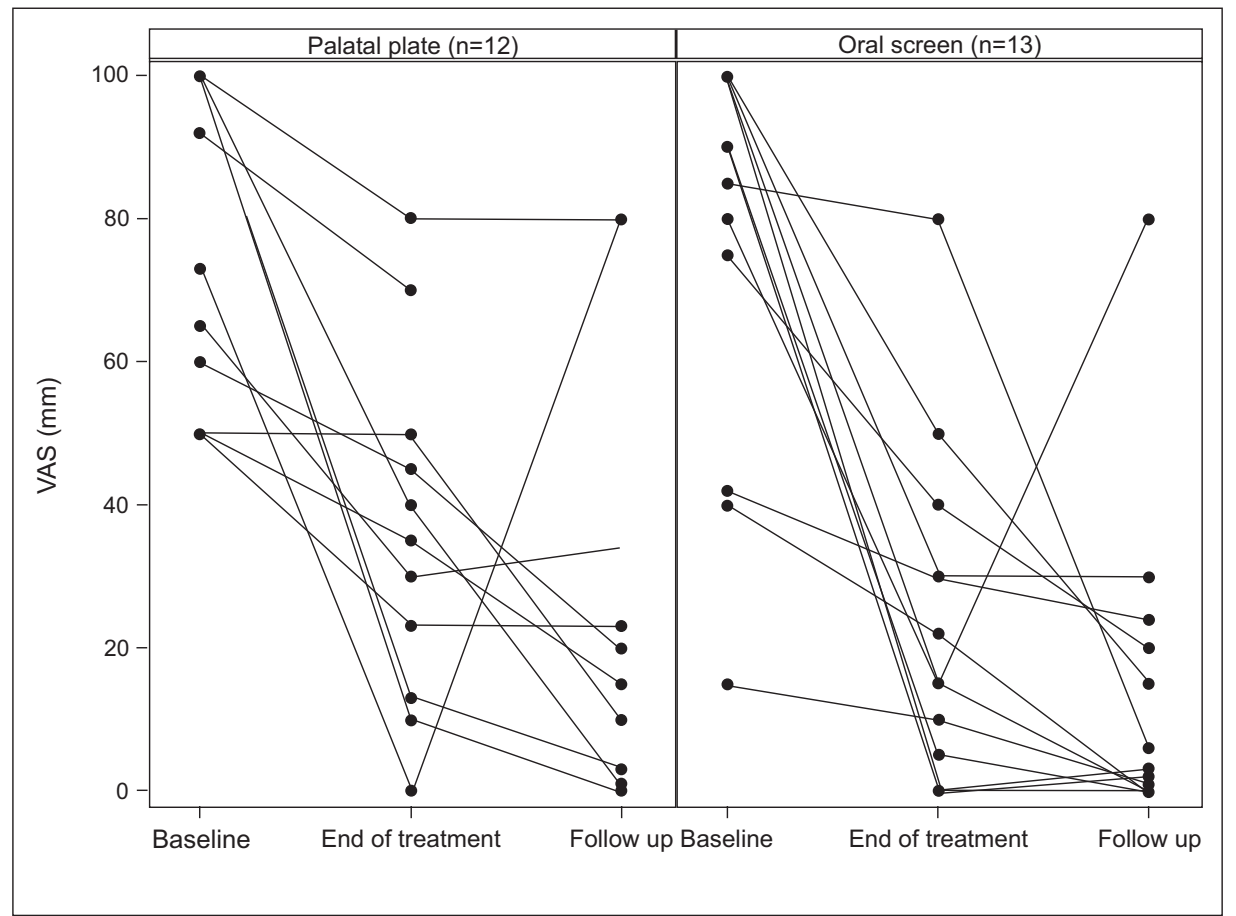

Fig. (3). Individual values on the visual analogue scale (VAS). Individual values on the VAS ( 0 mm $=$ normal; $100=$ severe disability) at baseline, end of treatment, and follow-up in stroke patients treated with a palatal plate (left) or with an oral screen (right).

stimulates the buccinator mechanism passively. Active training of striated muscles is superior to passive training for improvement of muscle strength. The PP training was predominantly of passive character $[14,15]$ whereas the OStraining was entirely active $[16,17]$. However, the subjective improvement did not differ significantly between the PP and OS training modalities.

OS training has even other advantages over PP training; an OS training session takes less than one minute and is less complicated than a PP training session requiring approximately half an hour to perform; individual fabrication of a PP presupposes access to a specialized dentist and a specialized dental technician. In contrast, a standardized OS can easily be delivered from a dental company and can easily be applied by various therapists for instance speech therapists, physiotherapists, and occupational therapists. In addition, the total cost for OS training is about $1 / 25^{\text {th }}$ that of PP training.
To our knowledge, studies have not yet been performed to determine if the training effect on post-stroke dysphagia can be maintained for a prolonged period of time. One of the reasons for the lack of late follow-up may be the difficulty in obtaining enough study patients, since the survival rate after stroke is rather low [29]. Dropout due to death in our study was approximately $50 \%$, which is similar to survival rates reported elsewhere [29]. Of the originally referred stroke afflicted dysphagia patients, only one-fifth were available for late follow-up, with losses due to death or repeat stroke.

The PP and the OS groups were indeed examined at two separate time periods. From start of the study the OS device was not available that precluded randomization of patients in the two groups. When the cheaper and more easily OStraining method was started in our clinic, PP training was precluded on ethical and economical grounds. It can be claimed that the two groups may have differed in some important ways thus preventing impartial evaluation. 
Table 3. Swallowing Capacity Test (SCT) in the "Early" and "Late" OS Groups

\begin{tabular}{|c|c|c|c|c|c|c|}
\hline Oral Screen (OS) Treatment & $\begin{array}{c}\text { Early }(n=7) \\
\text { Median SCT (Range) }\end{array}$ & p-value & $\begin{array}{l}\text { \% Normal } \\
\text { SCT }\end{array}$ & $\begin{array}{c}\text { Late }(n=7) \\
\text { Median SCT (Range) }\end{array}$ & p-Value & $\begin{array}{l}\text { \% Normal } \\
\text { SCT }\end{array}$ \\
\hline Baseline & $0.0(0.0-7.1)$ & & $0 \%$ & $4.8(0.0-10.0)$ & & $14 \%$ \\
\hline End of treatment & $12.0(4.0-13.8)$ & & $57 \%$ & $13.3(9.4-18.8)$ & & $86 \%$ \\
\hline Follow-up & $9.7(5.5-11.5)$ & & $43 \%$ & $21.7(12.1-27.7)$ & & $100 \%$ \\
\hline Change in SCT from baseline to end of treatment & $7.4(3.1-13.8)$ & $<0.02$ & & $9.6(4.2-15.4)$ & $<0.02$ & \\
\hline Change in SCT from baseline to follow-up & $8.1(4.1-11.0)$ & $<0.02$ & & $15.9(12.1-19.9)$ & $<0.02$ & \\
\hline Change in SCT from end of treatment to follow-up & $-0.6(-6.5-7.0)$ & $<0.81$ & & $6.3(-3.3-15.3)$ & $<0.05$ & \\
\hline
\end{tabular}

Median SCT (ml/s) values and ranges in the "Early" and "Late" treated OS groups, the percentage of patients with normal SCT before treatment (baseline), at end of treatment, and at follow-up, and median changes of SCT between the three examination periods (Wilcoxon's signed rank test). $=$ decrease.

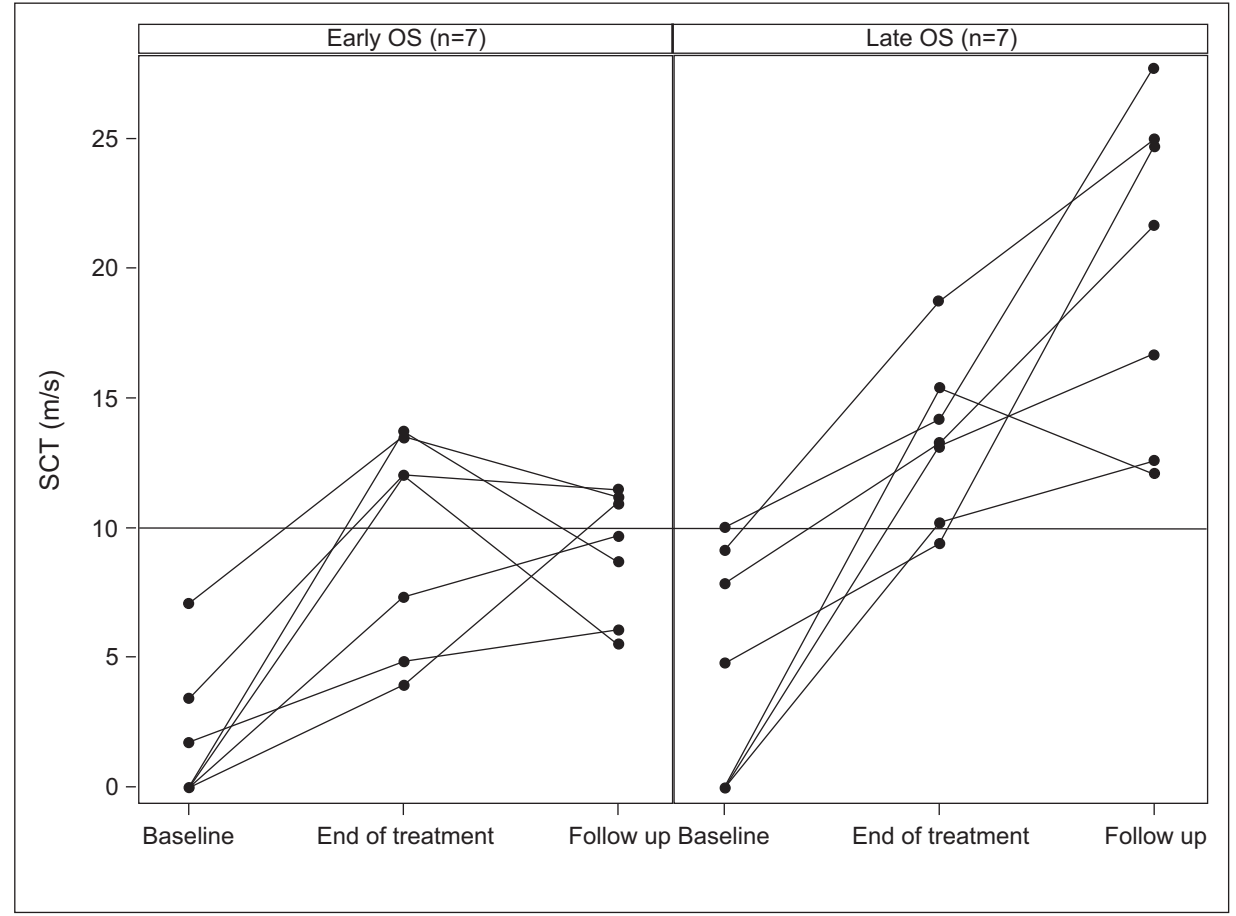

Fig. (4). Individual values on the swallowing capacity test (SCT) in the "Early" and "Late" OS group. Individual values of SCT (ml/s) at baseline, end of treatment, and follow-up in patients treated with an oral screen either "Early" (left) or "Late" (right) after stroke.

However, several objections were addressed at entry of the OS-study. The PP and OS groups had the same training length of 13-weeks. SCT and VAS were identically performed before start and after end of treatment. The two investigators were the same throughout all investigations. The PP and OS training started always at home, never in the hospital. The objective and subjective swallowing capacity did not differ at baseline between the PP and OS patients. However, the length of the period between end of treatment and late follow-up differed slightly between the groups but did not have any influence on longstanding training effect. Training compliance was optimal in both groups and could reasonably not have had any influence on the difference of training results. A possible confounding factor is that the median length of the time period between stroke attack and start of training was several months longer in the PP group than in the OS group. Most patients started the study with an almost complete inability to swallow water, even if a very long time had elapsed since the stroke. It was not any difference in training outcome between the OS group with a short and the OS group with a long time between stroke event and entry to the study. It is therefore very unlikely that spontaneous remission of swallowing capacity had taken place before the start of training.

\section{CONCLUSION}

The outcome of OS training on SCT in patients with stroke-related dysphagia seems to be superior to PP training. The improvement as assessed with VAS did not differ between the two groups. Training with PP or OS gives a longstanding improvement of SCT and VAS.

\section{CONFLICT OF INTEREST}

The authors report no conflict of interest. The authors alone are responsible for the content and writing of the paper. 
Table 4. Visual Analogue Scale (VAS) in the "Early" and "Late" OS Groups

\begin{tabular}{|c|c|c|c|c|}
\hline Oral Screen (OS) Treatment & Early $(n=7)$ Median VAS (Range) & p-Value & Late $(n=7)$ Median VAS (Range) & p-Value \\
\hline Baseline & $100(42-100)$ & & $80(15-100)$ & \\
\hline End of treatment & $30(0-80)$ & & $10(0-40)$ & \\
\hline Follow-up & $15(0-80)$ & & $0(0-20)$ & \\
\hline Change in VAS from baseline to end of treatment & $-70(-100--5)$ & $<0.02$ & $-65(-100--5)$ & $<0.02$ \\
\hline Change in VAS from baseline to follow-up & $-79(-97--12)$ & $<0.02$ & $-80(-100--14)$ & $<0.02$ \\
\hline Change in VAS from end of treatment to follow-up & $-5(-74-65)$ & $<0.438$ & $-9(-22-2)$ & $<0.125$ \\
\hline
\end{tabular}

VAS (mm) in the "Early" and "Late" treated oral screen (OS) group before treatment (baseline), at end of treatment and at follow-up, and changes in median VAS between the different test occasions (Wilcoxon's signed rank test). Statistical differences $=$ p-value, $-=$ decrease.

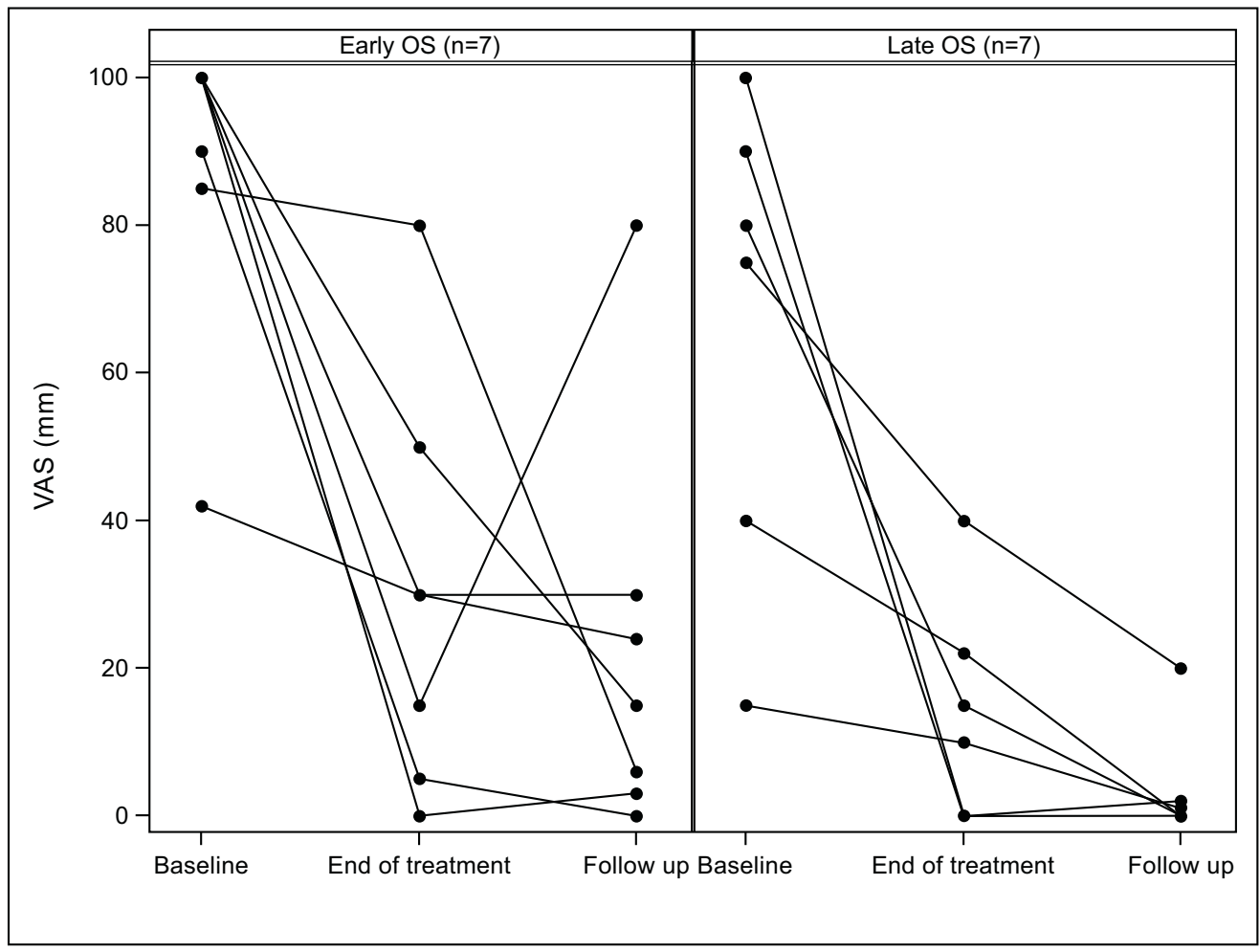

Fig. (5). Individual values on the visual analogue scale (VAS) in the "Early" and "Late" OS groups. Individual values on the VAS (0 $\mathrm{mm}=$ normal; $100=$ severe disability) at baseline, end of treatment, and follow-up in patients treated with an OS either "Early" (left) or "Late" (right) after stroke.

\section{ACKNOWLEDGEMENTS}

This work was supported by grants from The Centre for Research \& Development, Uppsala University/Gävleborg County Council, Sweden, The Council for Regional Research in Uppsala and Örebro region, and the Capio Research Foundation, Sweden. Many thanks go to Margaretha Olgarsson, Pt, Speech \& Swallowing Centre, Department of ENT, Hudiksvall Hospital, for invaluable help with patient care. Biostatistician Lisa Wernroth, Uppsala Clinical Research Centre (UCR), was involved from the outset in planning the study design and was responsible for statistical evaluation.

\section{REFERENCES}

[1] Martino R, Foley N, Bhogal S, Diamant N, Speechley M, Teasell R. Dysphagia after stroke: incidence, diagnosis, and pulmonary complications. Stroke 2005; 36: 2756-63.
[2] Mann G, Hankey GJ, Cameron D. Swallowing disorders following acute stroke Prevalence and diagnostic accuracy. Cerebrovasc Dis 2000; 10: 380-6.

[3] Martino R, Pron G, Diamant N. Screening for oropharyngeal dysphagia in stroke: insufficient evidence for guidelines. Dysphagia 2000; 15: 19-30.

[4] Mann G, Hankey G, Cameron D. Swallowing function after stroke. Prognosis and prognostic factors at 6 months. Stroke 1999; 30: $744-8$.

[5] Paciaroni M, Mazzotta G, Corea F, et al. Dysphagia following stroke. Eur Neurol 2004; 51: 162-7.

[6] Yildiz N, Ertekin C, Ozdemirkiran T, et al. Corticonuclear innervation to facial muscles in normal controls and in patients with central facial paresis. J Neurol 2005; 252: 429-32.

[7] Broadley S, Cheek A, Salonikis S, et al. Predicting Prolonged Dysphagia in Acute Stroke: The Royal Adelaide Prognostic Index for Dysphagic Stroke (RAPIDS). Dysphagia 2005; 20: 303-10.

[8] Smithard DG, O'Neill PA, England RE, et al. The natural history of dysphagia following a stroke. Dysphagia 1997; 12: 188-93. 
[9] Camaby G, Hankey G, Pizzi J. Behavioural intervention for dysphagia in acute stroke: a randomized controlled trial. Lancet Neurol 2006; 5: 31-7.

[10] Logemann JA. Evaluation and Treatment of Swallowing Disorder. $2^{\text {nd }}$ ed. Austin, TX: PRO-ED 1998; pp. 91-95, 194,195, 205-23.

[11] Teasell R, Foley N, Fisher J, Finestone H. The incidence, management, and complications of dysphagia in patients with medullary strokes admitted to a rehabilitation unit. Dysphagia 2002; 17: 115-20.

[12] Lin LC, Wang SC, Chen SH, Wang TG, Chen MY, Wu SC. Efficacy of swallowing training for residents following stroke. Adv Nurs 2003; 44: 469-78.

[13] Runions S, Rodrique N, White C. Practice on an acute stroke unit after implementation of a decision-making algorithm for dietary management of dysphagia. J Neurosci Nurs 2004; 13: 49-54.

[14] Castillo Morales RC, Brondo JJ, Haberstock B. Die orofaziale Regulationstherapie. $1^{\text {st }}$ ed. München: Richard Pflaum Verlag GmbH \& Co 1991; pp. 21-188.

[15] Hägg M, Larsson B. Effects of motor and sensory stimulation in stroke patients with long-lasting dysphagia. Dysphagia 2004; 19: 219-30.

[16] Hägg M, Anniko M. Influence of lip force on swallowing capacity in stroke patients and in healthy subjects. Acta Otolaryngol 2010; 130: $1204-8$.

[17] Hägg M, Anniko M. Lip muscle training in stroke patients with dysphagia. Acta Otolaryngol 2008; 128 (9): 1027-33.

[18] Robbins J, Butler SG, Daniels SK, et al. Swallowing and dysphagia rehabilitation: translating principles of neural plasticity into clinically oriented evidence. J Speech Lang Hear Res 2008; 51: 276-300.
[19] Robbins J, Kays SA, Gangnon RE, et al. The effects of lingual exercise in stroke patients with dysphagia. Arch Phys Med Rehabil 2007; 88: 150-8.

[20] Kays S, Robbins J. Effects of sensorimotor exercise on swallowing outcomes relative to age and age-related disease. Semin Speech Lang 2006; 27: 245-59.

[21] Hägg M. Olgarsson M. Anniko M. Reliable Lip force measurement in healthy controls and in patients with stroke: A methodological study. Dysphagia 2008; 2: 291-6.

[22] Perkins RE, Blanton PL, Biggs N. Electromyographic analysis of the "buccinator mechanism" in human beings. J Dent Res 1977; 56: 783-94.

[23] Logemann JA. Screening, diagnosis and management of neurogenic dysphagia. Semin Neurol 1996; 16: 319-27.

[24] Logemann JA. Swallowing disorders caused by neurologic lesions from which some recovery can be anticipated. Evaluation and treatment of swallowing disorders. $2^{\text {nd }}$ ed. Austin, TX: Pro-Ed Inc.1998; pp. 307-26.

[25] Hoyer H, Limbrock GJ. Orofacial regulation therapy in children with Down syndrome, using the methods and appliances of Castillo Morales. ASDC J Dent Child 1990; 57: 442-4.

[26] Chusid JK. Correlative neuroanatomy \& functional neurology. Los Altos, CA: Lange Medical Publication 1985.

[27] Nathadwarawala KM, Nicklin J, Wiles CM. A timed test of swallowing capacity for neurological patients. J Neurol Neurosurg Psychiatr 1992; 55: 822-5.

[28] Kalyanee M, Nathadwarawala KM, McGroary A, Wiles CM. Swallowing in neurological outpatients. Use of a timed test. Dysphagia 1994; 9: 120-9.

[29] Brønnum-Hansen H, Davidsen M, Thorvaldsen P. Long-term survival and causes of death after stroke. Stroke 2001; 32: 2131-6.

(C) Hägg and Tibbling; Licensee Bentham Open.

This is an open access article licensed under the terms of the Creative Commons Attribution Non-Commercial License (http: //creativecommons.org/licenses/by$\mathrm{nc} / 3.0 /$ ) which permits unrestricted, non-commercial use, distribution and reproduction in any medium, provided the work is properly cited. 